\title{
La Francophonie ivoirienne
}

Enjeux politiques et socioculturels

\section{Alain Laurent Aboa Abia}

\section{(2) OpenEdition}

Journals

Édition électronique

URL : https://journals.openedition.org/dhfles/123

DOI : $10.4000 /$ dhfles. 123

ISSN : 2221-4038

Éditeur

Société Internationale pour l'Histoire du Français Langue Étrangère ou Seconde

Édition imprimée

Date de publication : 1 janvier 2008

Pagination : 163-178

ISSN : 0992-7654

Référence électronique

Alain Laurent Aboa Abia, «La Francophonie ivoirienne », Documents pour l'histoire du français langue étrangère ou seconde [En ligne], 40/41 | 2008, mis en ligne le 17 décembre 2010, consulté le 27 mai 2021. URL : http://journals.openedition.org/dhfles/123 ; DOI : https://doi.org/10.4000/dhfles.123

Ce document a été généré automatiquement le 27 mai 2021

(c) SIHFLES 


\title{
La Francophonie ivoirienne
}

\author{
Enjeux politiques et socioculturels
}

\author{
Alain Laurent Aboa Abia
}

\section{Introduction}

1 L'avènement de la francophonie a sensiblement modifié la donne linguistique dans plusieurs pays africains au sud du Sahara. L'évolution de la langue française, dans cette partie du continent, se fait à un rythme tel que certains analystes comme Chaudenson (2000: 80) n'hésitent pas à penser que «l'avenir du français en Europe et dans le monde, se jouera hors d'Europe et principalement dans le sud. »

2 En effet, venue d'ailleurs, dernière venue dans le landerneau linguistique et culturel de l'Afrique contemporaine, c'est pourtant la langue française qui occupe le terrain, assure l'enseignement et dit le discours officiel. Il est vrai qu'elle fut introduite dans les pays africains par la force des baïonnettes, mais pour Cheikh Hamidou Kane (1977) :

Si elle y demeure et s'y maintient dans la position privilégiée qui est la sienne, c'est du fait du choix des Africains. Deux faits témoignent de ce choix qui sont d'une part le mouvement vers l'école française de ce peuple, mouvement dont l'ampleur a dépassé la capacité d'accueil et d'adaptation de l'école, d'autre part, le maintien du français à la fonction et à la dignité de langue officielle après l'accession des pays à la souveraineté internationale ${ }^{1}$.

Dans les milieux où, généralement, aucune langue locale ne sert d'outil de communication interethnique (principalement dans les zones urbaines) la langue française a même acquis le statut de langue véhiculaire. C'est le cas de la Côte d'Ivoire où dans la communication courante, le français s'est développé et particularisé2. On ne saurait en conclure évidemment que le français est parlé et compris par l'ensemble de la population ivoirienne. Seulement, l'usage local $\mathrm{du}$ français s'est quelque peu différencié en raison de l'environnement naturel et culturel, de l'influence des parlers locaux, des divers substrats linguistiques et ... de la volonté politique. Comment s'est faite l'évolution de la francophonie ivoirienne? 
Nous nous proposons d'analyser cette interrogation en mettant en relief, dans la première partie de cet article, les facteurs historiques de cette francophonie. Dans la deuxième partie, nous essayerons d'appréhender, à la lumière de la politique linguistique de la Côte d'Ivoire après l'indépendance, les enjeux politiques et socioculturels liés à la francophonie ivoirienne. Dans la troisième partie, nous verrons comment le français est parlé dans les différentes couches de la société.

\section{Les facteurs historiques de l'implantation de la langue française en Côte d'Ivoire}

5 L'implantation de la langue française en Côte d'Ivoire remonte au XIX ${ }^{\mathrm{e}}$ siècle avec l'ère coloniale. Dès son installation et pour faire face au vide culturel qu'il croit trouver, le colonisateur va établir sa culture. Il s'en suit alors une péjoration des langues locales. Prenant prétexte de ce que ces langues ne possèdent pas d'écriture, sont donc supposées incapables de traduire les sciences et de véhiculer des notions modernes, le colonisateur va montrer que l'on a tout à gagner, à apprendre et à parler sa langue. Devant les vertus et les sortilèges que l'on prête à cette langue, il se produit un attrait fasciné sur les populations. Très vite, des groupes d'intérêts vont se constituer. Et c'est autour de ces groupes que va se jouer la bataille linguistique ${ }^{3}$.

En effet, il se dégage, selon Louis-Jean Calvet (1974:60) :

une classe de collaborateurs qui va, par nécessité ou par intérêt, utiliser la langue du colonisateur: les commerçants. Parallèlement et pour les mêmes raisons de nécessité et d'intérêts, un autre groupe va apprendre cette langue. Ce groupe est le résultat d'une situation économique. La langue française est adoptée par ceux qui, sur place, sont proches du pouvoir ou le représentent et ceux qui traitent avec lui.

7 La langue maternelle est alors perçue comme une non-valeur en face de la néo-valeur que représente la langue du colonisateur. Comme l'écrit Alexandre Pierre (1971) :

Les interprètes coloniaux formaient une nouvelle élite, non tribale ou plus exactement supra-tribale, définie et, dans une certaine mesure, hiérarchisée, en fonction de sa maîtrise de la langue coloniale qui donnait un quasi-monopole d'accès au secteur moderne. [...] Grâce à la maîtrise du français, des descendants captifs se sont trouvés un jour libérés du travail physique. Le français a ainsi contribué à l'édification de nouvelles barrières sociales qui ne coïncident plus avec les barrières léguées par la tradition.

Conscient de ce que le système colonial a besoin d'un terreau fertile, face aux enjeux politiques et économiques, le colonisateur va imposer sa langue pour servir de bréviaire à son idéologie $e^{4}$. Ainsi, tout un apparat juridique est mis en place. A travers des décrets, des choix politiques et une planification scolaire, l'administration coloniale assure l'implantation de la langue française sur le territoire ivoirien. L'école est utilisée en conformité avec un projet idéologique qui vise à assurer l'hégémonie de la langue française.

De ce fait, de façon progressive, l'enseignement, durant l'époque coloniale en Côte d'Ivoire, sera organisé par les différents Gouverneurs qui vont se succéder à la tête de l'administration. L'un d'entre eux, le Gouverneur Mouttet, durant sa régence, va élaborer, en décembre 1877, un programme des écoles élémentaires de la colonie de Côte d'Ivoire.

Ce programme comprenait l'enseignement moral et civique, la lecture et l'écriture, la langue française, le calcul et le système métrique, l'histoire et la géographie de la 
France et de l'Afrique, des leçons de choses avec les premières notions scientifiques, l'agriculture, le dessin, le travail manuel, la musique vocale et des exercices de gymnastique. Il y avait trente heures de classe par semaine. La plus grande partie du temps était consacrée à l'étude du français. Le souci de l'heure étant de former, pour les besoins des services administratifs, des hommes ayant, en plus d'une connaissance passable de la langue française, quelques notions d'arithmétique et de système métrique. » (Sosso $1984: 46)$

10 En réalité, les écoles avaient non seulement pour but d'établir un contact entre les peuples attardés dont on venait de conquérir les territoires et les hommes venus de France, mais également de rassurer les indigènes sur les intentions des Français et d'établir entre eux et ces derniers, des rapports de sympathie. Ces écoles devaient permettre aux indigènes d'évoluer dans leur propre milieu, tout en transformant le pays sans en déraciner les habitants.

11 D'ailleurs, comme aimait à le dire le Gouverneur général William Ponty, les écoles n'étaient pas des "écoles à diplômes", car elles faisaient abstraction de tout enseignement théorique ou livresque pour ne s'intéresser qu'à l'utile, au profitable, au pratique et surtout à l'immédiat. Elles ne s'adressaient pas à une élite. L'important était la quantité des élèves.

L'enseignement en Côte d'Ivoire, depuis les origines jusqu'en 1954, ne s'est pas articulé facilement. Ce fut une œuvre de maints efforts. Dans ce territoire riche, mais aux populations d'ethnies diverses et souvent instables aux communications longtemps difficiles, cet art qui consiste à montrer, à faire découvrir, s'est organisé tardivement. Et jusqu'en 1954, son développement était encore limité car en 1910, par exemple, la Côte d'Ivoire ne comptait que 40 écoles alors qu'en Gold Coast ${ }^{5}$, le nombre d'écoles s'élevait à 260. » (Sosso 1984 : 78)

12 Cependant, un peu plus tard, de 1951 à 1954, sa progression s'accentue car le taux global de scolarisation a été de $7 \%$ en $1951 ; 8 \%$ en $1952 ; 12 \%$ en 1953 et $15 \%$ en 1954 (R. Papy, 1954). Progressivement, l'école va promouvoir la langue française auprès des indigènes et consolider, par ricochet, les bases du système colonial. Mais tout ceci procède d'un calcul politique.

13 En effet, en présentant la langue française comme la langue de la civilisation, la langue d'avenir, le colonisateur se cultive un mythe, amène les populations à lui faire allégeance et fait ainsi tourner les rapports de force à son avantage. C'est d'ailleurs ce que révèle Meister lorsqu'il écrit : « derrière la phraséologie humaniste, c'est un calcul politique que la réalité oblige à faire ».

14 L'enjeu, c'est de conforter la suprématie de la langue française sur l'échiquier ivoirien et plus généralement d'établir la domination du système colonial. Pour ce faire, dans certains de leurs travaux, des analystes vont théoriser et donner des arguments linguistiques à la politique coloniale. Une théorisation que Maurice Houis (1971) qualifie de « caution savante à la politique coloniale». C'est le cas du dénommé Davesne dont l'ouvrage paru à Saint-Louis en 1933 a un titre assez évocateur : La langue française, langue de civilisation en Afrique occidentale française ${ }^{6}$.

\section{La politique linguistique ivoirienne après I'Indépendance}

15 La domination de la langue française dans le paysage linguistique ivoirien n'a pas disparu avec l'accession du pays à l'indépendance le 7 août 1960. Les autorités 
ivoiriennes vont poursuivre la politique linguistique mise en œuvre durant la colonisation?

16 Cela se traduit par l'élévation de la langue française au rang de langue officielle. Ce statut est formellement établi par la première constitution de la Côte d'Ivoire qui dispose dans son article $1^{\mathrm{er}}$ : «la langue officielle est le français $»^{8}$. Ce choix politique consacre la domination légale de la langue française dans tous les domaines de la vie publique :

Le français est la langue de toutes les institutions politiques, de la jurisprudence, du système scolaire à tous les niveaux, de la police, de l'armée et des médias. (Kube $2005: 76)$

17 Curieusement, cette constitution ne fait aucune mention des langues nationales (une soixantaine environ).

18 Si la politique linguistique de la Côte d'Ivoire confère à la langue française un statut officiel et institutionnalisé, il n'y a pas non plus de politique de péjoration et de dévalorisation des langues locales :

Celles-ci continuent d'être parlées à la radio et à la télévision, dans certaines émissions ou occasionnellement dans les publicités. La loi permet même l'utilisation d'une langue ivoirienne aux justiciables qui éprouvent des difficultés à s'exprimer en français. » (Kube 2005)

19 Le premier document officiel qui fait référence aux langues nationales est la loi portant réforme de l'enseignement adoptée le 16 août 1977 par le parlement ivoirien. Deux articles de cette loi consacrent l'introduction prochaine des langues ivoiriennes dans l'enseignement. L'article 67 stipule: «l'introduction des langues ivoiriennes dans l'enseignement officiel est conçue comme un facteur d'unité nationale et de revalorisation du patrimoine ivoirien ", et l'article 68 ajoute : «l'Institut de linguistique appliquée est chargé de préparer l'introduction des langues nationales dans l'enseignement, notamment par leur description ».

20 Seulement, les textes définissant les modalités d'application de la loi de 1977 n'ont pas suivi. Malgré cela, les chercheurs de l'Institut de linguistique appliquée se sont évertués à réaliser des descriptions scientifiques complètes, à préparer du matériel didactique et à engager des expérimentations (Lafage 1996). La volonté politique, elle, tarde à suivre le mouvement. La posture adoptée par les autorités s'explique essentiellement par des enjeux politiques et socioculturels.

\section{Les enjeux politiques}

21 La raison centrale avancée par les autorités pour justifier le choix de la langue française comme langue officielle est la conservation de la cohésion sociale et de l'unité nationale.

En effet, il est vrai que le français est au départ l'outil de la colonisation, cependant, c'est cette langue qui a permis de former des élites et d'entrer aisément dans les relations internationales. Pour les autorités ivoiriennes, il n'est donc pas question d'abandonner un outil aussi précieux.

Cette langue doit au contraire se répandre dans tous les domaines de la société ivoirienne et se montrer indispensable pour avoir accès à un statut élevé. Elle doit être maîtrisée par les intellectuels, les enseignants, les cadres industriels et commerciaux pour que la Côte d'Ivoire soit entendue à l'échelle internationale.» (Boutin 1998 : 18) 
Déjà cinq ans avant l'indépendance de la Côte d'Ivoire, Félix Houphouët Boigny, à la veille de sa nomination comme ministre délégué à la Présidence du Conseil, propose la création d'une communauté franco-africaine, association égalitaire entre la France métropolitaine et ses territoires d'outre-mer.

Notre vœu ardent est que toutes les familles spirituelles françaises comprennent que le Rassemblement Démocratique Africain est tourné vers l'ensemble du peuple français avec le désir de bâtir avec lui une communauté durable où les inévitables querelles de famille ne nuiront pas à la loyauté de vivre ensemble. » (Cité d'après Boutin 1998:5)

24

Le choix de la langue française comme langue officielle après l'accession de la Côte d'Ivoire à l'indépendance, répond à un enjeu éminemment politique : se positionner sur l'échiquier politique international. Et pour atteindre cet objectif, les autorités n'ont pas trouvé mieux que le français, une langue internationale. «On ne se pose pas la question de savoir si le français, langue officielle, est la meilleure façon d'assurer la promotion collective des peuples et leur développement. » (Calvet 1974).

Dans l'esprit des dirigeants, le français permettrait d'entrer directement dans les grands courants d'échanges internationaux et d'entretenir des relations privilégiées avec les pays qui comptent sur la scène internationale. De même, « ce choix permettrait d'envisager des regroupements régionaux entre pays voisins à un moment où florissait l'idéologie panafricaine ». (Duponchel 1974)

Même si l'argument politique n'apparaît pas clairement dans le discours officiel, il n'en demeure pas moins l'élément central de la politique linguistique des autorités ivoiriennes. La volonté de s'ouvrir vers le monde extérieur et de créer des conditions de développement économique est l'une des raisons essentielles du choix de la langue française comme langue officielle?

D'ailleurs, s'exprimant à une tribune des Nations Unies, le Président de l'Assemblée nationale de l'époque disait :

Je dois toutefois à la vérité de dire qu'en ce qui concerne mon pays, l'adoption du français, par l'article premier de notre Constitution, a été sans doute l'un des facteurs d'unité qui ont favorisé l'aboutissement heureux et si rapide de l'œuvre de construction nationale dont son Excellence le président Félix Houphouët-Boigny avait fait un des premiers thèmes de son action. Le français, librement accepté par nous, a été un facteur de cohésion à l'intérieur de la Côte d'Ivoire où il a favorisé le regroupement de nos quelque cent ethnies ${ }^{10}$. (Cité d'après Leclerc $2002: 2$ )

La relation entre l'idée de développement économique de la Côte d'Ivoire après l'indépendance et la politique linguistique est ici évidente. «En optant pour la langue française, on pensait atteindre rapidement le niveau de développement des pays du nord. » (Kube 2005)

Et c'est ce que confirment ces propos de Jules Hié Néa, ancien ministre de la Culture : « il ne faut pas oublier que la Côte d'Ivoire a choisi un développement ouvert sur le monde extérieur: la nécessité d'utiliser une langue internationale s'impose par de telles considérations. » (Cité d'après Leclerc $2002: 2$ )

Outre les enjeux politiques qui ont milité en faveur du choix de la langue française comme langue officielle, il y a également des enjeux socioculturels. 


\section{Les enjeux socioculturels}

31 n'appartient à aucune ethnie, elle était plus à même de favoriser la paix sociale et l'unité nationale. Dans un contexte ambiant particulier, où aucune des soixante langues locales ne sert véritablement de véhiculaire interethnique, la langue française apparaît comme l'unique médium susceptible de démêler l'écheveau des contradictions internes, de neutraliser les particularismes locaux, «de fondre des groupes ethniques souvent rivaux en une seule nation et de canaliser les tendances centrifuges de certains »(Duponchel 1974).«Le français représente l'unité nationale au-delà de toute rivalité interne, étant lui-même en dehors de celle-ci dès le départ. » (Boutin 1998)

imaginaire linguistique et culturel des dirigeants ivoiriens, la force du sentiment interne que les populations ont de leurs cultures ne suffit plus à préserver ces dernières à présent que ces populations entrent dans le cycle du progrès au sein d'un monde devenu un.

L'ouverture vers une langue internationale (comme la langue française), dotée de l'outil précieux qu'est l'écriture, s'avère donc nécessaire pour codifier les lois et conserver les croyances. De plus, la langue française a été préférée aux langues nationales parce qu'elle est jugée plus à même de traduire les arts et les sciences et d'ouvrir l'accès à la technologie et à la civilisation moderne. C'est d'ailleurs pour cette raison que cette langue est devenue, en Côte d'Ivoire, un facteur de promotion sociale. Elle est donc une importante clé sociale, confère des pouvoirs exorbitants et ceux qui en profitent n'ont, bien entendu, aucune envie de les perdre. Même la nouvelle élite gargarisée par l'idéologie panafricaine, en dehors de toute propagande particulariste de caractère racial ou ethnique, reconnaît que la connaissance de la langue française est un facteur de prestige et d'ascension sociale.

D'ailleurs, aucune élite ivoirienne actuelle ne doit sa situation à la maîtrise de sa langue maternelle ni à sa connaissance des traditions. Ainsi apparaît l'ambiguïté des prises de position des intellectuels africains. Malgré les déclarations vengeresses, bien peu sont prêts à abandonner, pour eux et surtout pour leurs enfants, la solide rente que constitue la possession du français. (Duponchel 1974)

\section{Les variétés de français en Côte d'Ivoire}

La plupart des recherches menées sur le français de Côte d'Ivoire font état de l'existence de trois variétés de français dans ce pays. Le français des élites (variété acrolectale), le français des lettrés (variété mésolectale) et le français des peu ou non lettrés (variété basilectale). L'usage de la langue française en Côte d'Ivoire s'est, au fil du temps, particularisé et différencié, au point qu'il est difficile, aujourd'hui, d'établir une définition exacte de ces différentes variétés de français.

Seulement, on constate désormais l'existence d'une multitude d'usages et de modes d'appropriation de la langue, influencée plus par le contexte de la pratique que par la couche socioculturelle dont le locuteur fait partie (Simard 1994).

Chaque locuteur possède plusieurs variétés du français qu'il utilise dans les situations de communication qu'il modifie de ce fait ${ }^{11}$. (Ploog $2002: 35$ ).

Kouadio Jérémie (1999: 301) pense cependant que la pratique actuelle du français en Côte d'Ivoire ne permet plus que la distinction de deux variétés : «le français local, la 
variété ivoirienne du français populaire ivoirien (FPI), la variété qui est apprise en dehors de l'école. Le français local ou français de Côte d'Ivoire inclut, par conséquent, la variété moyenne et la variété acrolectale ».

Le français populaire ivoirien s'est constitué tout d'abord à Abidjan. Il apparaît comme une variété non pas achevée, mais en cours d'évolution, aussi bien au niveau des locuteurs, qui sont des apprenants du français à plusieurs stades, qu'au niveau social. Si en 1971, les chercheurs étaient réticents à parler de français populaire ivoirien et qu'ils le traitaient surtout comme relevant d'une ou de deux phases d'apprentissage du français, on constate aujourd'hui que ce français est attesté dans tout le pays (Boutin 1998). De même, Jean-Louis Hattiger (1981) qui a entrepris des recherches sur la morphosyntaxe du français populaire ivoirien pense que «cette variété de français constitue un phénomène unique en Afrique francophone ».

Le français populaire ivoirien est parlé par des individus que l'on pourrait considérer comme membres de la classe moyenne basse. Il s'agit notamment de scolarisés de l'enseignement primaire, d'alphabétisés en français et d'illettrés parlant français. La période de scolarisation - dans les cas où le français a été appris à l'école - a été trop brève pour que subsiste longtemps la référence à la norme académique. (Lafage 1978)

S'agissant des deux autres variétés de français parlées en Côte d'Ivoire: la variété acrolectale (variété supérieure parlée par l'élite ivoirienne) et la variété mésolectale (variété moyenne parlée par les lettrés de l'enseignement secondaire et les cadres subalternes de l'administration ivoirienne), Kouadio (1999: 301) dit avoir la conviction que "ces deux variétés se confondent et que les traits qui les séparent sont moins nombreux que ceux qui les rapprochent ».Il estime donc que la variété mésolectale et la variété acrolectale pourraient être regroupées sous l'appellation «français de Côte d'Ivoire ». Ces deux variétés obéissent, de plus en plus, aux règles implicites de la norme locale.

41 Dans une analyse descriptive et contrastive, Kouadio (1999: 301) présente «certaines tendances syntaxiques du français écrit en Côte d'Ivoire en l'opposant de façon implicite ou explicite au français central». Par exemple, au niveau de la détermination nominale, il note que l'usage du déterminant en français de Côte d'Ivoire connaît une restructuration dont les points saillants sont ${ }^{12}$ :

- Absence de déterminant ou déterminant zéro :

1. (E1) : Entre bicyclette et poste radio, je préfère bicyclette.

- Emploi de l'article défini en lieu et place du possessif :

1. (E2) : La jeunesse sportive nous fait honte, nous perdons d'ailleurs le temps en parlant d'elle.

- Substitution de l'article défini à l'article partitif :

1. (E3): On le fait cuire avec l'huile.

- Substitution de l'article indéfini à l'article défini :

1. (E4) : Il ne laisse pas une moindre tache sur ses vêtements.

En fait, on peut considérer qu'à des degrés différents d'acquisition de la langue officielle, correspond un système de hiérarchisation dans la société ivoirienne. La catégorisation s'établit en termes de telle ou telle variété de français. (Lafage 1978) 
Dans le milieu des années 1980, l'apparition du nouchi comme variété la plus récente du français ivoirien a été expliquée par la fonction identitaire que remplit ce moyen de communication. Selon Sabine Kube (2005: 41) :

Utilisé au début comme code secret par des jeunes de la rue, le nouchi a été vite adopté par les élèves et étudiants, ce qui a réduit son caractère cryptique. Aujourd'hui, la pratique du nouchi est assez différente de celle observée en 1990, année de la "découverte scientifique» de ce phénomène linguistique. Présenté au début comme une langue ésotérique parlée par les jeunes délinquants, le nouchi tend à «se véhiculariser ". D'après Boutin (2002), on peut supposer que toute la population ivoirienne, l'élite intellectuelle comprise, a au moins des connaissances passives du nouchi. Elle pense, par ailleurs, que l'existence de l'appellation «français nouchi » rapproche celui-ci du français et le fait entrer dans le « français pluriel ».

Il faut toutefois préciser que le nouchi se différencie du français populaire et des autres variétés principalement par son vocabulaire caractérisé, selon Kouadio Jérémie (1990) par:

50 - Des emprunts massifs aux langues ivoiriennes (en particulier au dioula et au baoulé) et non ivoiriennes :
1. (E5) : Soutra (du dioula « aider $»)^{13}$
2. (E6) : Blo (du baoulé « exagérer », « se vanter de ... »)
3. C'est blo (C'est exagéré)
4. (E7) : Enjailler (de l'anglais enjoy « s'amuser »)

51 - Resémantisation de certains termes français qui deviennent alors polysémiques :

1. (E8) : Elle l'a drap en classe (Elle l'a insulté devant la classe)

2. (E9) : Je suis en drap de ça (Je suis au courant de ça)

3. (E10) : Ya pas drap (Pas de problème)

52 - Création de nombreux mots :

1. (E11) : La kraya (la faim)

2. (E12) : Badou (manger)

3. (E13) : Etre tchass (être fauché)

4. (E14) : Tchapa (parler, raconter)

5. (E15) : Gbaher (sécher les cours)

\section{Conclusion}

53 De l'époque coloniale au lendemain des indépendances, la situation de la langue française a considérablement évolué en Côte d'Ivoire. D'abord utilisée pour servir de bréviaire à la politique coloniale, cette langue va ensuite, par la volonté des autorités ivoiriennes, arborer l'étendard de la nouvelle société. L'évolution du français et son appropriation par les Ivoiriens sont dues à trois facteurs : le caractère de nécessité du français, l'absence d'un véhiculaire africain et le développement économique.

De ce fait, souverain dans un paysage linguistique ivoirien où aucune des 64 ethnies n'a émergé pour devenir « la langue de la majorité, du fait que l'idéal d'unité nationale et de respect des autres peuples est fortement présent chez tous, malgré les difficultés 
inhérentes à un tel processus d'identification » (Boutin 1998 : 6), le français connaît, en Côte d'Ivoire, un cas particulier d'appropriation.

\section{BIBLIOGRAPHIE}

ALEXANDRE, Pierre (1971). Multilingualism, Current Trends in Linguistics, Volume 7, La HayeParis, Mouton.

BOUTIN, Béatrice (1998). Quelques variantes syntaxiques du français en Côte d'Ivoire, mémoire de DEA, université Stendhal-Grenoble 3.

- (2002). Description de la variation : études transformationnelles des phrases du français de Côte d'Ivoire, thèse de doctorat, université Stendhal-Grenoble 3.

CALVET, Louis-Jean (1974). Linguistique et colonialisme, petit traité de glottophagie, Paris, Payot.

CHAUDENSON, Robert (2000). Mondialisation : la langue française a-t-elle encore un avenir ?, Paris, L'Harmattan.

DUPONCHEL, Laurent (1974). Le français en Côte d'Ivoire, au Dahomey et au Togo, Abidjan, ILA.

HATTIGER, Jean-Louis (1981). Morphosyntaxe du groupe nominal dans un corpus de français populaire d'Abidjan, thèse de doctorat, Strasbourg.

HOUIS, Maurice (1971). Anthropologie linguistique de l'Afrique noire, Paris, PUF.

KANE, Cheikh Hamidou (1977). « Langue française et identité culturelle sénégalaise ».

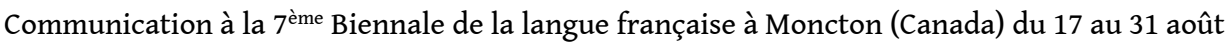
1977. Publiée par Le Soleil dans la rubrique « Arts et Lettres » des 14 et 21 octobre 1977.

KOUADIO, N. Jérémie (1999). «Quelques traits morphosyntaxiques du français en Côte d'Ivoire », in Langues, volume 2.

KUBE, Sabine (2005). La francophonie vécue en Côte d'Ivoire, Paris, L'Harmattan.

LAFAGE, Suzanne (1978). « Observatoire du français contemporain en Côte d'Ivoire : rôle et place du français populaire dans le continuum langues africaines / français de Côte d'Ivoire ", in Cahier Ivoirien de Recherches Linguistiques, ILA, UNACI, Abidjan.

LAFAGE, Suzanne (1996). « La Côte d'Ivoire, une appropriation nationale du français ? », in Le français dans l'espace francophone, tome 2, Paris.

LECLERC, Jacques (2002). « Côte d'Ivoire », in L'aménagement linguistique dans le monde, Québec, TLFQ (Université Laval).

MEISTER, Albert (1973). Alphabétisation et développement, Paris, Éditions Anthropos.

PAPY, R. (1954). «L'enseignement en Côte d'Ivoire », in Cahiers Charles de Foucauld, numéro sur la Côte d'Ivoire (France), vol. 1.

PLOOG, Katja (2002). Le français à Abidjan : pour une approche syntaxique du non-standard, Paris, CNRS Editions. 
SIMARD, Yves (1994). «Les français de Côte d'Ivoire ", in Le français en Afrique Noire, faits d'appropriation, Langue française, $\mathrm{n}^{\circ} 104$.

SOSSO, Léonard (1984). L'enseignement en Côte d'Ivoire depuis les origines jusqu'en 1954, tome 1, Abidjan.

\section{NOTES}

1. Cheikh Hamidou Kane (1977) explique aussi l'hégémonie de la langue fran-çaise dans plusieurs pays d'Afrique au sud du Sahara par le fait que cette langue possède une écriture. Pour lui, l'outil qu'est l'écriture, ses vertus et ses sortilèges ne pouvait pas manquer d'exercer sur les peuples africains de tradition orale un attrait fasciné.

2. La rapidité avec laquelle s'est développé le français en Côte d'Ivoire tient à la situation linguistique de ce pays. Boutin (1998) révèle qu'en Côte d'Ivoire, le français n'a jamais été en situation de diglossie avec une seule langue locale dominante, c'est-à-dire de complémentarité selon les interactions langagières.

3. Louis-Jean Calvet (1974) précise que le bilinguisme (franco-africain) vécu durant la période coloniale ne concerne qu'un petit groupe d'individus: l'immense majorité de la population demeure monolingue. De façon générale, l'extension linguistique est, selon lui, à sens unique. Il ne s'agit pas de vases communicants mais d'injection.

4. Au plan linguistique, le colonialisme institue, selon Calvet (1974), un champ d'exclusion d'une langue (la langue dominée) des sphères du pouvoir, exclusion des locuteurs de cette langue (de ceux qui n'ont pas appris la langue dominante) de ces mêmes sphères.

5. Gold Coast : aujourd'hui ce pays porte le nom de Ghana, nom qui lui a été donné après son accession à l'indépendance.

6. Cette œuvre montre bien que la pensée coloniale transforme les rapports de différence en rapport de supériorité. Selon Calvet (1974), lorsque le colonialisme aura besoin de justifier sa propre entreprise, il lui suffira de puiser dans cette théorie de la supériorité du colonisateur et d'y ajouter un grain d'humanisme.

7. Comme le relève, à juste titre, Boutin (2002: 43), Félix Houphouët Boigny ne s'est jamais prononcé sur le français et n'a jamais fait connaître publiquement ses raisons du choix de cette langue. Seulement, en rapprochant certains faits concernant l'indépendance de la Côte d'Ivoire ou l'attitude de Félix Houphouët Boigny face à la langue française, l'on peut percevoir en quoi consistait le choix du français par le premier Président ivoirien.

8. Constitution de la République de Côte d'Ivoire, novembre 1960.

9. Le français continue de jouer un rôle important dans la vie quotidienne des Ivoiriens et symbolise l'accès à la modernité. Perçu naguère comme langue de l'aliénation, il tend à devenir véhicule d'une libération.

10. La conviction des autorités ivoiriennes est que la préservation de la paix sociale passe nécessairement par l'utilisation du français, une langue neutre et étrangère aux contradictions internes.

11. Sabine Kube (2002: 40) pense que cette situation est loin de faciliter une recherche linguistique. Un avis que partage Ploog (2002:12) qui soutient que c'est la raison pour laquelle la Côte d'Ivoire et en particulier Abidjan ne font plus partie aujourd'hui des régions de l'espace francophone les plus étudiées.

12. Les exemples que nous citons sont extraits de "Quelques traits morpho-syntaxiques du français écrit en Côte d'Ivoire » de Kouadio Jérémie (1999) dans Langues, volume 2.

13. Nous avons relevé ces exemples dans «la francophonie vécue en Côte d'Ivoire » de Sabine Kube (2005). 


\section{RÉSUMÉS}

Dans le panorama linguistique et culturel de l'Afrique contemporaine, le terrain n'est pas seulement habité par les langues natives. La langue française, héritage de la colonisation, a été élevée à la dignité de langue officielle, après l'accession des pays à la souveraineté nationale et internationale. Son choix n'a suscité alors aucune opposition notable, ni chez les élites, ni dans la population. De ce fait même, cette langue acquiert une position privilégiée. C'est ainsi qu'en Côte d'Ivoire, l'avènement de la francophonie a été brandi comme un moyen puissant de neutraliser les particularismes locaux et les tendances centrifuges de certains. Les autorités de ce pays n'ont eu de cesse de répéter que, parce cette langue n'appartient à aucune ethnie, elle était plus à même de préserver l'unité nationale. Cette idée entretenue dans l'imaginaire linguistique des dirigeants ivoiriens va donc entrâner la relégation des langues locales à la périphérie des enjeux politiques nationaux et conforter, par ricochet, l'hégémonie de la francophonie sur l'échiquier sociolinguistique ivoirien. Dès lors, dans toutes les occasions de la vie sociale, c'est la langue française qui dit la loi, transmet le savoir moderne, véhicule les échanges les plus importants. Au fond, l'histoire de la francophonie ivoirienne est l'histoire d'un choix justifié par l'entreprise coloniale et dont les facteurs n'ont pas disparu malgré l'accession de ce pays à l'indépendance.

In the linguistic and cultural panorama of contemporary Africa, the land is not only inhabited by the native languages. The French language (heritage of colonization), was high with the dignity of official language after the accession of the french-speaking countries to national and international sovereignty. Its choice then caused no opposition with the elites nor the population. Therefore, the language acquires a privileged position. Thus in Ivory Coast, the advent of the francophonie was held up as a powerful means of neutralising local particularisms and the centrifugal tendencies of some. The political authorities of this country continue its usage because French language does not belong to any ethnic group, as it consequently preserves the national unity. This idea is maintained by the linguistic leaders of Ivory Coast. This will incorporate the relegation of the local languages to the periphery of the national and political stakes. This in turn will consolidate the hegemony of the francophonie on the chessboard sociolinguistic of the Ivory Coast. Consequently, in all occasions of social life, it is the French language which communicates the law, transmits modern knowledge and conveys the most significant exchanges. Fundamentally, the history of the francophonie of the Ivory coast still continues to be a choice justified by the colonial company and whose factors did not disappear in spite of the accession of this country to independence.

\section{INDEX}

Keywords : francophony, independence, politic, sociocultural, stakes

Mots-clés : enjeux, francophonie, indépendance, politique, socioculturel

\section{AUTEUR}

\section{ALAIN LAURENT ABOA ABIA}

Université de Cocody, Abidjan, Côte d'Ivoire

aboaalainlaurent@yahoo.fr 